\title{
A FORMULA FOR ANALYTIC SEPARATION CAPACITY
}

\author{
By TAKAFUmi Murai
}

\section{Introduction.}

For a compact set $E$ in the complex plane $C, H^{\infty}\left(E^{c}\right)$ denotes the Banach space of bounded analytic functions in $E^{c}=\boldsymbol{C} \cup\{\infty\}-E$ with supremum norm $\|\cdot\|_{H^{\infty}}$. The analytic capacity of $E$ is defined by

$$
\gamma(E)=\sup \left\{\left|f^{\prime}(\infty)\right| ; f \in H^{\infty}\left(E^{c}\right),\|f\|_{H^{\infty}} \leqq 1\right\},
$$

where $f^{\prime}(\infty)=\lim _{z \rightarrow \infty} z\{f(\infty)-f(z)\} \quad[5$, p. 6]. The analytic capacity of $E$ at $a \in \boldsymbol{C}-E$ is defined by

$$
c_{E}(a)=\sup \left\{\left|f^{\prime}(a)\right| ; f \in H^{\infty}\left(E^{c}\right),\|f\|_{H^{\infty}} \leqq 1\right\} \quad[10, \text { Chap III }] .
$$

These set-functions play various important roles in the study of bounded analytic functions. As a general set-function, we define the analytic separation capacity of $E$ at $a, b \in E^{c}, a \neq b$ by

$$
\delta(E, a, b)=\sup \left\{|f(b)-f(a)| ; f \in H^{\infty}\left(E^{c}\right),\|f\|_{H^{\infty}} \leqq 1\right\} \quad[6,7] .
$$

We easily see that

$$
\lim _{b \rightarrow \infty}|b| \delta(E, \infty, b)=\gamma(E), \quad \lim _{b \rightarrow a} \frac{\delta(E, a, b)}{|b-a|}=c_{E}(a)
$$

and that $\delta(E, a, b)>0$ if and only if $\gamma(E)>0$. Hence $\delta(E, a, b)$ is applicable to study $\delta(E)$ and $c_{E}(a)$, and this set-function is important to investigate bounded analytic functions which separate a and $b$. The purpose of this note is to show a formula for $\delta(E, a, b)$. Let $\mathcal{A}$ denote the totality of finite unions of mutually disjoint analytic arcs. Here an arc is analytic, if it is a portion of an analytic Jordan curve. For $E \in \mathcal{A}, L^{2}(E)$ denotes the $L^{2}$ space of functions on $E$ with respect to the length element $|d z|$. For a bounded function $g$ on $E, M_{g}$ denotes the multiplier $f \in L^{2}(E) \rightarrow g f \in L^{2}(E)$, and $I d_{E}$ denotes the identity operator. The operator $H_{E}$ from $L^{2}(E)$ to itself is defined by

$$
\begin{aligned}
H_{E} h(z) & =\frac{1}{\pi} p \cdot v \cdot \int_{E} \frac{h}{\zeta-z}|d \zeta| \\
& =\frac{1}{\pi} \lim _{\varepsilon \downarrow 0} \int_{|\zeta-z|>\varepsilon, \zeta \in E} \frac{h}{\zeta-z}|d \zeta| \quad\left(h \in L^{2}(E)\right),
\end{aligned}
$$

Received Otober 20, 1989; Revised December 22, 1989. 
and the operator $\bar{H}_{E}$ is defined by $\bar{H}_{E} h=\overline{H_{E}} \bar{h}$. For the computation of $\delta(E, a, b)$, we may assume that $E \in \mathcal{A}$. (See Proposition 9.) Since $\delta(E, a, b)$ is conformally invariant, we may assume that $a, b \neq \infty$. In this note, we shall establish

Theorem. For $E \in \mathcal{A}$ and $a, b \in C-E, a \neq b$,

$$
\delta(E, a, b)=\frac{|b-a|}{\operatorname{det} A_{0}}\left(a_{23} a_{31}-a_{21} a_{33}\right),
$$

where $A_{0}$ is a (3,3)-matrix defined by

$$
\begin{aligned}
& a_{11}=1+\frac{1}{\pi} \int_{E} \frac{1}{z-a} U_{0}\left(g_{0}^{-1} \bar{H}_{E} g_{0}\right)|d z|, \\
& a_{12}=\frac{1}{\pi} \int_{E} \frac{1}{z-a} U_{0}\left(\frac{1}{\overline{\cdot-a}} g_{0}^{-1}\right)|d z|, \\
& a_{13}=\frac{1}{\pi} \int_{E} \frac{1}{z-a} U_{0}\left(\frac{1}{\overline{(\cdot-a)(\cdot-b)}} g_{0}^{-1}\right)|d z|, \\
& a_{21}=\frac{1}{\pi} \int_{E}\left\{1+H_{E} U_{0}\left(g_{0}^{-1} \bar{H}_{E} g_{0}\right)\right\} g_{0}|d z|, \\
& a_{22}=\frac{1}{\pi} \int_{E} H_{E} U_{0}\left(\frac{1}{\overline{\cdot-a}} g_{0}^{-1}\right) \cdot g_{0}|d z|-1, \\
& a_{23}=\frac{1}{\pi} \int_{E} H_{E} U_{0}\left(\frac{1}{(\cdot-a)(\cdot-b)} g_{0}^{-1}\right) \cdot g_{0}|d z|, \\
& a_{31}=\frac{1}{\pi} \int_{E} \frac{1}{(z-a)(z-b)} U_{0}\left(g_{0}^{-1} \bar{H}_{E} g_{0}\right)|d z|, \\
& a_{32}=\frac{1}{\pi} \int_{E} \frac{1}{(z-a)(z-b)} U_{0}\left(\frac{1}{\cos ^{-a}} g_{0}^{-1}\right)|d z|, \\
& a_{33}=\frac{1}{\pi} \int_{E} \frac{1}{(z-a)(z-b)} U_{0}\left(\frac{1}{(\cdot-a)(\cdot-b)} g_{0}^{-1}\right)|d z|, \\
& g_{0}(z)=\frac{1}{|(z-a)(z-b)|}, \quad U_{0}=\left(I d_{E}-M_{1 / g_{0}} \bar{H}_{E} M_{g_{0}} H_{E}\right)^{-1} .
\end{aligned}
$$

As application of our theorem, we shall deduce

Corollary 1. For $E \subset \boldsymbol{R}$ and $a \in \boldsymbol{C}-E, \operatorname{Im} a \neq 0$,

$$
\delta(E, a, \bar{a})=2 \tan \left\{\frac{1}{4}\left|\operatorname{Im} \int_{E} \frac{d x}{x-a}\right|\right\},
$$

where $\operatorname{Im} \zeta$ is the imaginary part of $\zeta$ and $\boldsymbol{R}$ is the real line.

COROllary 2. For $E \subset \boldsymbol{R}$ and $a, b \in \boldsymbol{R}-E$ contained in a component of 
$\boldsymbol{R} \cup\{\infty\}-E$,

$$
\delta(E, a, b)=2 \tanh \left\{\frac{1}{8}\left|\int_{E} \frac{(b-a) d x}{(x-a)(x-b)}\right|\right\}
$$

COROLlarary 3. If $E$ is a compact set on the unit circle $\boldsymbol{T}$, then $\delta(E, 0, \infty)=2 \tan (|E| / 8)$, where $|\cdot|$ is the 1-dimensional Lebesgue measure.

Pommerenke [11] shows that

(1) $\gamma(E)=|E| / 4 \quad(E \subset \boldsymbol{R})$.

The method given in [9] shows that

(2) $\gamma(E)=\sin (|E| / 4) \quad(E \subset \boldsymbol{T})$.

(See [2], also.) Our corollaries correspond to (1) and (2). In the section 2, we shall give the proof of our theorem. In the section 3 , we shall show a proposition concerning the Hilbert transform, which plays an important role in the computation of capacities induced from the Hilbert transform. In the section 4, we shall deduce Corollary 1 from our theorem. Our method is not short, however, this shows a method to construct the extremum pair $\left(f_{0}, \phi_{0}\right)$ (cf. Lemmas 4 and 6 ) and this is applicable to compute various capacities. We shall give another proof of Corollary 1 also; once the extremum pair $\left(f_{0}, \phi_{0}\right)$ is found, a short proof is possible. The proof of Corollaries 2 and 3 will be given in this section. In the last section, we shall show some applications of our method. The author expresses his thanks to Professors Suita, Shiba, Yamada and Masumoto for their variable comments about $\delta(E, a, b)$.

\section{Proof of Theorem.}

In this section, we give the proof of our theorem. Evidently, $\delta(E, a, b)$ is conformally invariant: $\delta(E, a, b)=\delta(F, f(a), f(b))$ if $f$ conformally maps $E^{c}$ onto $F^{c}$. If $a$ and $b$ belong to different components of $E^{c}$, then $\delta(E, a, b)=2$. Hence it is essential to study the case where $a$ and $b$ are contained in a component of $E^{c}$. We may assume that this component contains $\infty$. Since $\delta(E, a, b)=$ $\delta(\partial E, a, b)$, we assume, throughout this note, that $E^{c}$ is connected. Let $\mathscr{I}$ denote the totality of finite unions of mutually disjoint analytic arcs and mutually disjoint compact sets bounded by analytic Jordan curves. For $E \in \mathscr{I}$ and $p \geqq 1, H^{p}\left(E^{c}\right)$ denotes the totality of analytic functions $f$ in $E^{c}$ such that $|f|^{p}$ is integrable on the boundary $\partial E$ of $E$ with respect to the length element $|d z|$. If a component $l$ of $E$ is an arc, then its boundary has two sides. Here are some lemmas necessary for the proof; Lemmas 4 and 5 are applications of the Ahlfors-Garabedian method [1], [4] to $\delta(E, a, b)$.

LEMMA 4 (Garabedian [4], Lax [7]). Let $E \in \mathscr{F}$ and $a, b \in \boldsymbol{C}-E, a \neq b$. 
Then there exists a pair $\left(f_{0}, \phi_{0}\right)$ of functions such that $f_{0} \in H^{\infty}\left(E^{c}\right), \phi_{0}$ is analytic in $E^{c}-\{a, b\}, \int_{\partial E}\left|\psi_{0}\right||d z|<\infty$,

(3) $\left\{\begin{array}{l}\phi_{0}(z)=-1 /(z-a)+\cdots \text { in a neighborhood of } a, \\ \phi_{0}(z)=1 /(z-b)+\cdots \text { in a neighborhood of } b, \\ \lim _{z \rightarrow \infty} z \psi_{0}(z)=0,\end{array}\right.$

(4) $\left|f_{0}\right|=1$ almost everywhere (a.e.) on $\partial E$,

(5) $\frac{1}{i} f_{0} \psi_{0} d z \geqq 0$ a.e. on $\partial E$,

where the orientation of $d z$ is chosen so that $E^{c}$ lies to the left. Moreover, this pair $\left(f_{0}, \psi_{0}\right)$ satisfies

(6) $\delta(E, a, b)=f_{0}(b)-f_{0}(a)=\frac{1}{2 \pi} \int_{\hat{o} E}\left|\psi_{0}\right||d z|$.

Garabeidan [4] shows an analogous lemma by using the Green's functions and harmonic measures, and Lax [7] shows this lemma by using the HahnBanach theorem. In this note, we give a sketch of a simplified proof which shows a relation between $f_{0}$ and $\phi_{0}$. Without loss of generality, we may assume that $E$ is bounded by analytic Jordan curves. Let

$$
\delta *(E, a, b)=\inf \left\{\frac{|b-a|}{2 \pi} \int_{\partial E}|\rho| g_{0}|d z| ; \rho \in H^{1}\left(E^{c}\right), \quad \rho(a)=\rho(b)=1\right\},
$$

where $g_{0}(z)=1 /|(z-a)(z-b)|$. There exists $\rho_{0} \in H^{1}\left(E^{c}\right), \rho_{0}(a)=\rho_{0}(b)=1$ which attains $\delta *(E, a, b)$. A variational method shows that

(7) $\int_{\partial E} \frac{\bar{\rho}_{0}}{\left|\rho_{0}\right|} \rho g_{0}|d z|=0$

for all $\rho \in H^{1}\left(E^{c}\right)$ satisfying $\rho(a)=\rho(b)=0$. Choosing a point $z_{0}$ in the interior of $E$, we put

$$
\left\{\begin{array}{l}
f_{0}(z)=\frac{1}{2 \pi(b-a)} \int_{\hat{\partial} E}\left\{\frac{(z-b)(\zeta-a)}{\zeta-z}-\frac{\left(z_{0}-b\right)(\zeta-a)}{\zeta-z_{0}}\right\} \frac{\bar{\rho}_{0}}{\left|\rho_{0}\right|} g_{0}|d \zeta|, \\
\phi_{0}(z)=\frac{b-a}{(z-a)(z-b)} \rho_{0}(z) .
\end{array}\right.
$$

Then (7) shows that

$$
f_{0}(z)=i \frac{(z-a)(z-b)}{(b-a)} \frac{\bar{\rho}_{0}}{\left|\rho_{0}\right|} g_{0} \frac{|d z|}{d z} \text { a. e. on } \partial E,
$$

which yields (3)-(5). Equality (6) is immediately deduced from (3)-(5). 
Lemma 5. Let $E, a, b, g_{0}$ and $\delta *(E, a, b)$ be the same as above and let

$$
\delta * *(E, a, b)=\inf \left\{\frac{|b-a|}{2 \pi} \int_{\partial E}|\phi|^{2} g_{0}|d z| ; \phi \in H^{2}\left(E^{c}\right), \quad \phi(a)=\phi(b)^{2}=1\right\} .
$$

Then

$$
\delta(E, a, b)=\delta *(E, a, b)=\delta * *(E, a, b) .
$$

This lemma is deduced from Garabedian's method [4]. In fact, we have easily $\delta(E, a, b)=\delta *(E, a, b) \leqq \delta * *(E, a, b)$. To prove $\delta *(E, a, b) \geqq \delta * *(E, a, b)$, we may assume that $E$ is bounded by analytic Jordan curves. Let $\rho_{0}$ be the function attaining $\delta *(E, a, b)$. Then (3)-(5) yield that $\sqrt{\rho_{0}}$ is single-valued, where a branch is chosen so that $\sqrt{\rho_{0}(a)}=1$. Putting $\phi_{0}=\sqrt{\rho_{0}}$, we obtain

$$
\delta *(E, a, b)=\frac{|b-a|}{2 \pi} \int_{\partial E}\left|\phi_{0}\right|^{2} g_{0}|d z| \geqq \delta * *(E, a, b) .
$$

Consequently, $\delta *(E, a, b)=\delta * *(E, a, b)$.

LEMMa 6. For $E \in \mathscr{F}$, the pair $\left(f_{0}, \phi_{0}\right)$ satısfyıng (3)-(5) is unique.

Proof. Let $\left(f_{0} *, \phi_{0} *\right)$ also satisfy (3)-(5). Since

$$
\delta(E, a, b)=f_{0} *(b)-f_{0} *(a)=\frac{1}{2 \pi \imath} \int_{\partial E} f_{0} * \psi_{0} d z \frac{1}{2 \pi}=\int_{\partial E}\left|\psi_{0}\right||d z|,
$$

we have $\frac{1}{\imath} f_{0} * \phi_{0} d z=\left|\phi_{0}\right||d z|=\frac{1}{\imath} f_{0} \phi_{0} d z$ a. e. on $\partial E$, and hence $f_{0} *=f_{0}$ a. e. on $\partial E$, which shows that $f_{0} *=f_{0}$. Let

(8) $\delta_{\varepsilon} * *(E, a, b)=\inf \left\{\frac{|b-a|}{2 \pi} \int_{\partial E}|\phi|^{2} g_{0}|d z| ; \phi \in H^{2}\left(E^{c}\right)\right.$,

$$
\phi(a)=\varepsilon \phi(b)=1\} \quad(\varepsilon= \pm)
$$

Then, by Fatou's lemma and the Pappos median line theorem, there uniquely exists $\phi_{\varepsilon} \in H^{2}\left(E^{c}\right)$ which attains $\delta_{\varepsilon} * *(E, a, b)(\varepsilon= \pm)$. Since

$$
\delta(E, a, b):=\delta * *(E, a, b)=\min \left\{\delta_{+} * *(E, a, b), \delta_{-} * *(E, a, b)\right\},
$$

the following four cases are possible for the pair $\left(\psi_{0}, \phi_{0} *\right)$ :

$$
\left(\phi_{+}^{2} g_{0} *, \phi_{+}^{2} g_{0} *\right), \quad\left(\phi_{-}^{2} g_{0} *, \phi_{-}^{2} g_{0} *\right), \quad\left(\phi_{+}^{2} g_{0} *, \phi_{-}^{2} g_{0} *\right), \quad\left(\phi_{-}^{2} g_{0} *, \phi_{+}^{2} g_{0} *\right),
$$

where $g_{0} *(z)=\frac{b-a}{(z-a)(z-b)}$. In the first two cases, we have $\phi_{0}=\phi_{0} *$. Suppose that $\left(\psi_{0}, \psi_{0} *\right)$ equals either the third pair or the last pair. Since

$$
\delta(E, a, b)=\frac{1}{2 \pi \imath} \int_{\partial E} f_{0}\left\{\frac{\phi_{0}+\psi_{0} *}{2}\right\} d z
$$


a pair $\left(f_{0},\left(\phi_{0}+\phi_{0} *\right) / 2\right)$ also satisfies (3)-(5). Thus $\sqrt{\left(\phi_{0}+\phi_{0} *\right) /\left(2 g_{0} *\right)}(=\phi *$, say) is single-valued and "either $\phi *=\phi_{+}$or $\phi *=\phi_{-}$". Hence either $\left(\phi_{0}+\phi_{0} *\right) / 2=\phi_{0}$ or $\left(\phi_{0}+\phi_{0} *\right) / 2=\phi_{0} *$. Consequently, $\phi_{0}=\phi_{0} *$. This completes the proof.

Lemma 7. Let $E, a, b$ be the same as in Lemma 4. Then $\delta(E, a, b)=$ $\delta_{+} * *(E, a, b)$, where $\delta_{+} * *(E, a, b)$ is the quantity defined by (8).

Proof. Fixing $a \in \boldsymbol{C}-E$, we put $W_{\varepsilon}=\left\{b \in \boldsymbol{C}-E \cup\{a\} ; \delta(E, a, b)=\boldsymbol{\delta}_{\varepsilon} * *(E, a, b)\right\}$ $(\varepsilon= \pm)$. Then Lemma 6 shows that $W_{+} \cup W_{-}=\boldsymbol{C}-E \cup\{a\}$ and $W_{+} \cap W_{-}=\varnothing$. We show that $W_{\varepsilon}(\varepsilon= \pm)$ are closed in $C-E \cup\{a\}$. Let $\left(b_{n}\right)_{n=1}^{\infty}$ be a convergent sequence in $W_{+}$such that $\lim _{n \rightarrow \infty} b_{n}\left(=b_{\infty}\right.$, say) belongs to $\boldsymbol{C}-E \cup\{a\}$. Note that $\delta\left(E, a, b_{\infty}\right)=\lim _{n \rightarrow \infty} \delta\left(E, a, b_{n}\right)$. There exists a sequence $\left(\phi_{n}\right)_{n=1}^{\infty}$ in $H^{2}\left(E^{c}\right)$ such that $\phi_{n}\left(b_{n}\right)=1$ and $\phi_{n}$ attains $\delta\left(E, a, b_{n}\right)(n \geqq 1)$. By an argument of a normal family and Fatou's lemma, there exists $\phi_{\infty} \in H^{2}\left(E^{c}\right), \phi_{\infty}\left(b_{\infty}\right)=1$ such that

$$
\liminf _{n \rightarrow \infty} \delta\left(E, a, b_{n}\right) \geqq \frac{\left|b_{\infty}-a\right|}{2 \pi} \int_{\partial E}\left|\phi_{\infty}\right|^{2} g_{0}|d z| .
$$

This shows that $\phi_{\infty}$ attains $\delta\left(E, a, b_{\infty}\right)$. Thus $b_{\infty} \in W_{+}$. In the same manner, we see that $W_{-}$is closed. This shows that either $W_{+}=\boldsymbol{C}-E \cup\{a\}$ or $W_{+}=\varnothing$. If $\phi_{0} \in H^{2}\left(E^{c}\right)$ attains $\delta(E, a, b)$, we have, by Schwarz's inequality and $\delta(E, a, b)$ $\leqq 2$,

$$
\begin{aligned}
\left|\phi_{0}(b)-\phi_{0}(a)\right| & \leqq \frac{|b-a|}{2 \pi} \int_{\partial E}\left|\phi_{0}\right| g_{0}|d z| \\
& \leqq \delta(E, a, b)^{1 / 2}\left\{\frac{|b-a|}{2 \pi} \int_{\partial E} g_{0}|d z|\right\}^{1 / 2} \\
& \leqq \sqrt{2}\left\{\frac{|b-a|}{2 \pi} \int_{\partial E} g_{0}|d z|\right\}^{1 / 2}
\end{aligned}
$$

This shows that $\phi_{0}(a)=\phi_{0}(b)$ if $b$ is sufficiently near to $a$, which implies $W_{+} \neq \varnothing$. Thus $W_{+}=\boldsymbol{C}-E \cup\{a\}$, i. e., $\delta(E, a, b)=\delta_{+} * *(E, a, b)$ for all $b \in \boldsymbol{C}-E \cup\{a\}$. This completes the proof.

For $h \in L^{2}(E)$, we write

$$
\mathcal{C}_{E} h(z)=\frac{1}{\pi} \int_{E} \frac{h}{\zeta-z}|d \zeta| \quad(z \in C-E) .
$$

For a pair $(c, h), c \in C, h \in L^{2}(E)$ and $a, b \in C-E, a \neq b$, we write

$$
\|(c, h)\|_{a, b}=\left(\frac{|b-a|}{\pi} \int_{E}\left\{\left|c+H_{E} h\right|^{2}+|h|^{2}\right\} g_{0}|d z|\right)^{1 / 2} \text {. }
$$

Using Lemma 7, we show

Lemma 8. For $E \in \mathcal{A}$ and $a, b \in C-E, a \neq b$,

$$
\delta(E, a, b)=\inf \|(c, h)\|_{a, b}^{2},
$$


where the infimum is taken over all pairs $(c, h), c \in \boldsymbol{C}, h \in L^{2}(E)$ such that $c+\mathcal{C}_{E} h(a)=c+\mathcal{C}_{E} h(b)=1$. Moreover, the pair $\left(c_{0}, h_{0}\right)$ which attains $\delta(E, a, b)$ is unique.

Proof. For any pair $(c, h), c \in C, h \in L^{2}(E)$, we have $c+\mathcal{C}_{E} h \in H^{2}\left(E^{c}\right)$. Conversely, for any $\phi \in H^{2}\left(E^{c}\right)$, there uniquely exists a pair $(c, h), c \in C, h \in L^{2}(E)$ such that $\phi=c+\mathcal{C}_{E} h$. We see that

$$
c+\mathcal{C}_{E} h(z)=c+H_{E} h(z)+i h(z) \frac{|d z|}{d z} \text { a. e. on } \partial E
$$

and $d z=-d z^{\prime}$ if $z, z^{\prime} \in \partial E, z \neq z^{\prime}$ and the projections of $z$ and $z^{\prime}$ to $E$ are identical. Hence a simple calculation shows that

$$
\frac{|b-a|}{2 \pi} \int_{\partial E}|\phi|^{2} g_{0}|d z|=\|(c, h)\|_{a, b}^{2} .
$$

Thus Lemma 7 yields the required equality. The unicity of the pair $\left(c_{0}, h_{0}\right)$ is also an immediate consequence of Lemma 7. This completes the proof.

We now give the proof of our theorem. Let $\left(c_{0}, h_{0}\right)$ be the pair in Lemma 8. A variational method shows that

$$
\text { (9) } \frac{1}{\pi} \int_{E}\left\{\left(c_{0}+H_{E} h_{0}\right) \overline{\left(c+H_{E} h\right)}+h_{0} \bar{h}\right\} g_{0}|d z|=0
$$

for all pairs $(c, h), c \in \boldsymbol{C}, h \in L^{2}(E)$ satisfying

$$
\text { (10) } c+\mathcal{C}_{E} h(a)=c+\mathcal{C}_{E} h(b)=0 \text {. }
$$

Condition (10) is rewritten as

$$
c=-\frac{1}{\pi} \int_{E} \frac{h}{z-a}|d z|, \quad \frac{1}{\pi} \int_{E} \frac{h}{(z-a)(z-b)}|d z|=0 .
$$

Let

$$
\text { (11) } p_{0}=\frac{1}{\pi} \int_{E}\left(c_{0}+H_{E} h_{0}\right) g_{0}|d z| \text {. }
$$

Then (9) shows that

$$
\begin{aligned}
0= & p_{0} \bar{c}+\frac{1}{\pi} \int_{E}\left\{-\bar{H}_{E}\left(\left(c_{0}+H_{E} h_{0}\right) g_{0}\right)+h_{0} g_{0}\right\} \bar{h}|d z| \\
= & \frac{1}{\pi} \int_{E}\left\{h_{0} g_{0}-\bar{H}_{E}\left(\left(c_{0}+H_{E} h_{0}\right) g_{0}\right)-\frac{p_{0}}{z-a}\right\} \bar{h}|d z| \\
= & \frac{1}{\pi} \int_{E}\left\{h_{0}-g_{0}^{-1} \bar{H}_{E}\left(\left(c_{0}+H_{E} h_{0}\right) g_{0}\right)\right. \\
& \left.\left.-p_{0} \frac{1}{z-a} g_{0}^{-1}\right\} \overline{(z-a)(z-b)} g_{0} \frac{h}{(z-a)(z-b)}\right\}|d z| .
\end{aligned}
$$


Since $h \in L^{2}(E)$ is arbitrary as long as $\int_{E} \frac{h}{(z-a)(z-b)}|d z|=0$, we obtain

$$
\left\{h_{0}-g_{0}^{-1} \bar{H}_{E}\left(\left(c_{0}+H_{E} h_{0}\right) g_{0}\right)-p_{0} \frac{1}{z-a} g_{0}^{-1}\right\} \overline{(z-a)(z-b)} g_{0}=q_{0}
$$

for some constant $q_{0}$. Since

$$
-\int_{E}\left(M_{1 / g_{0}} \bar{H}_{E} M_{g_{0}} H_{E} h\right) \bar{h} g_{0}|d z|=\int_{E}\left|H_{E} h\right|^{2} g_{0}|d z| \geqq 0 \quad\left(h \in L^{2}(E)\right),
$$

$\left(I d_{E}-M_{1 / g_{0}} \bar{H}_{E} M_{g_{0}} H_{E}\right)$ is invertible, i. e., $U_{0}$ exists. Hence (12) shows that

(13) $h_{0}=c_{0} U_{0}\left(g_{0}^{-1} \bar{H}_{E} g_{0}\right)+p_{0} U_{0}\left(\frac{1}{\overline{\cdot-a}} g_{0}^{-1}\right)+q_{0} U_{0}\left(\frac{1}{(\cdot-a)(\cdot-b)} g_{0}^{-1}\right)$.

Substituing the conditions $c_{0}+\mathcal{C}_{E} h_{0}(a)=1,(11)$ and $\frac{1}{\pi} \int_{E} \frac{1}{(z-a)(z-b)} h_{0}|d z|=0$ by (13), we obtain

$$
A_{0}\left(\begin{array}{l}
c_{0} \\
p_{0} \\
q_{0}
\end{array}\right)=\left(\begin{array}{lll}
a_{11}, & a_{12}, & a_{13} \\
a_{21}, & a_{22}, & a_{23} \\
a_{31}, & a_{32}, & a_{33}
\end{array}\right)\left(\begin{array}{l}
c_{0} \\
p_{0} \\
q_{0}
\end{array}\right)=\left(\begin{array}{l}
1 \\
0 \\
0
\end{array}\right),
$$

where $A_{0}$ is the matrix in the assertion of our theorem. The matrix $A_{0}$ is invertible. In fact, if ${ }^{t}\left(c_{0} *, p_{0} *, q_{0} *\right)$ satisfies (14), we define $h_{0} *$ by (13) with respect to ${ }^{t}\left(c_{0} *, p_{0} *, q_{0} *\right)$. Then $\left(c_{0} *, h_{0} *\right)$ satisfies (9) for all pairs $(c, h), c \in \boldsymbol{C}$, $h \in L^{2}(E)$ satisfying (10), which yields that

$$
\left\|\left(c_{0} *+c, h_{0} *+h\right)\right\|_{a, b}^{2}=\left\|\left(c_{0} *, h_{0} *\right)\right\|_{a, b}^{2}+\|(c, h)\|_{a, b}^{2}
$$

for all pairs $(c, h)$ satisfying (10). This shows that $\left(c_{0} *, h_{0} *\right)$ attains $\delta(E, a, b)$. Thus the unicity gives that $\left(c_{0} *, h_{0} *\right)=\left(c_{0}, h_{0}\right)$. We have

$$
\begin{aligned}
0= & \left(I d_{E}-M_{1 / g_{0}} \bar{H}_{E} M_{g_{0}} H_{E}\right)\left(h_{0}-h_{0} *\right) \\
& =\left(c_{0}-c_{0} *\right) g_{0}^{-1} \bar{H}_{E} g_{0}+\left(p_{0}-p_{0} *\right) \frac{1}{z-a} g_{0}^{-1}+\left(q_{0}-q_{0} *\right) \frac{1}{(z-a)(z-b)} g_{0}^{-1} \\
& =\left(p_{0}-p_{0} *\right) \frac{1}{z-a} g_{0}^{-1}+\left(q_{0}-q_{0} *\right) \frac{1}{(z-a)(z-b)} g_{0}^{-1},
\end{aligned}
$$

and hence $\left(p_{0}-p_{0} *\right) \overline{(z-b)}+\left(q_{0}-q_{0} *\right)=0$ on $E$, which shows that $p_{0}=p_{0} *$ and $q_{0}=q_{0} *$. Thus ${ }^{t}\left(c_{0} *, p_{0} *, q_{0} *\right)={ }^{t}\left(c_{0}, p_{0}, q_{0}\right)$. Since the solution of (14) is unique. $A_{0}$ is invertible.

Since a pair $\left(c_{0}-1, h_{0}\right)$ satisfies $(10)$, we have

(15) $\delta(E, a, b)=\left\|\left(c_{0}, h_{0}\right)\right\|_{a, b}^{2}=\frac{|b-a|}{\pi} \int_{E}\left(c_{0}+H_{E} h_{0}\right) g_{0}|d z|=|b-a| p_{0}$. 
Hence Cramer's formula yields that

$$
\delta(E, a, b)=|b-a| p_{0}=\frac{|b-a|}{\operatorname{det} A_{0}}\left|\begin{array}{l}
a_{11}, 1, a_{13} \\
a_{21}, 0, a_{23} \\
a_{31}, 0, a_{33}
\end{array}\right|=\frac{|b-a|}{\operatorname{det} A_{0}}\left(a_{23} a_{31}-a_{21} a_{33}\right),
$$

Thus the required equality holds. This completes the proof.

Last, we note

Proposition 9. For a compact set $E$ in $C$ and $a, b \in C-E, a \neq b$, there exists a sequence $\left(E_{n}\right)_{n=1}^{\infty}$ in $A$ such that

$$
\delta(E, a, b)=\lim _{n \rightarrow 0} \delta\left(E_{n}, a, b\right) .
$$

Proof. There exists a decreasing sequence $\left(F_{m}\right)_{m=1}^{\infty}$ of compact sets bounded by mutually disjoint analytic Jordan curves such that $E=\cap_{m=1}^{\infty} F_{m}$. Then an argument on a normal family shows that $\delta(E, a, b)=\lim _{m \rightarrow \infty} \delta\left(F_{m}, a, b\right)$. Hence, from the beginning, we may assume that $E$ is bounded by mutually disjoint analytic Jordan curves. We express $\partial E$ as a union of Jordan curves: $\partial E=$ $\bigcup_{k=1}^{m} l_{k}$. Choosing a point $z_{k}$ on each $l_{k}(1 \leqq k \leqq m)$, we define

$$
E_{n}=\bigcup_{k=1}^{m}\left\{z \in l_{k} ;\left|z-z_{k}\right| \geqq 1 / n\right\} \quad(n \geqq 1) .
$$

Since $\left(E_{n}\right)_{n=1}^{\infty}$ is increasing and $E_{n} \subset E$, we see that $\lim _{n \rightarrow \infty} \delta\left(E_{n}, a, b\right)$ ( $=\delta_{0}$, say) exists and $\delta_{0} \leqq \delta(E, a, b)$. There exists $\phi_{n} \in H^{2}\left(E_{n}^{c}\right), \phi_{n}(a)=\phi_{n}(b)=1$ such that

$$
\delta\left(E_{n}, a, b\right)=\frac{|b-a|}{2 \pi} \int_{\partial E_{n}}\left|\phi_{n}\right|^{2} g_{0}|d z| .
$$

Let $\lambda_{n}=\partial E \backslash \partial E_{n}(n \geqq 1)$. Since

$$
\begin{aligned}
\phi_{n}(z) & =\phi_{n}(\infty)+\frac{1}{2 \pi i} \int_{\partial E_{n}} \frac{1}{\zeta-z} \phi_{n} d \zeta \\
& =\phi_{n}(a)-\frac{1}{2 \pi i} \int_{\partial E_{n}} \frac{1}{\zeta-a}\left\{\phi_{n}(\zeta)-\phi_{n}(a)\right\} d \zeta+\frac{1}{2 \pi i} \int_{\partial E_{n}} \frac{1}{\zeta-z} \phi_{n} d \zeta \\
& =1-\frac{1}{2 \pi i} \int_{\partial E_{n}} \frac{1}{\zeta-a} \phi_{n} d \zeta+\frac{1}{2 \pi i} \int_{\partial E_{n}} \frac{1}{\zeta-z} \phi_{n} d \zeta \quad\left(z \in \lambda_{n}\right),
\end{aligned}
$$

we have

$$
\frac{|b-a|}{2 \pi} \int_{\lambda_{n}}\left|\phi_{n}\right|^{2} g_{0}|d z| \leqq C_{0}
$$

for some constant $C_{0}$ independent of $n$, and hence

$$
\frac{|b-a|}{2 \pi} \int_{\partial E}\left|\phi_{n}\right|^{2} g_{0}|d z| \leqq \delta_{0}+C_{0} \quad(n \geqq 1),
$$


i. e., $\left(\phi_{n}\right)_{n=1}^{\infty}$ is bounded in the $L^{2}$ space of functions on $\partial E$ with respect to $g_{0}|d z|$. Let $\phi_{\infty}$ be a weak star cluster point of $\left(\phi_{n}\right)_{n=1}^{\infty}$. Then $\phi_{\infty} \in H^{2}\left(E^{c}\right)$ and $\phi_{\infty}(a)=\phi_{\infty}(b)=1$. For any compact set $K$ in $\partial E-\cup\left\{z_{k} ; 1 \leqq k \leqq m\right\}$, we have $\frac{|b-a|}{2 \pi} \int_{K}\left|\phi_{n}\right|^{2} g_{0}|d z| \leqq \delta_{0}$ as long as $K \subset \partial E_{n}$. Letting $n$ tend to infinity, we obtain $\frac{|b-a|}{2 \pi} \int_{K}\left|\phi_{\infty}\right|^{2} g_{0}|d z| \leqq \delta_{0}$. Since $K$ is arbitrary, this inequality holds with $K$ replaced by $\partial E$. Thus $\delta(E, a, b) \leqq \delta_{0}$. This completes the proof.

\section{A proposition for $H_{E}(E \subset \boldsymbol{R})$.}

Throughout this section, we assume that $E \in \mathcal{A}, E \subset \boldsymbol{R}$. In this case, $H_{E}$ is called the Hilbert transform on $L^{2}(E)$. Let $\chi \in L^{2}(E)$ denote the constant function taking only 1 . We inductively define a sequence $\left(H_{E}^{n}\right)_{n=0}^{\infty}$ of operators from $L^{2}(E)$ to itself by $H_{E}^{0}=I d_{E}, H_{E}^{n}=H_{E} H_{E}^{n-1}(n \geqq 1)$. Note that the norm of $H_{E}^{n}$ is less than or equal to $1(n \geqq 0)$. For $t \in \boldsymbol{R},|t|<1$ and $h \in L^{2}(E)$, we define a function $h_{t} \in L^{2}(E)$ by

$$
h_{t}=\sum_{n=0}^{\infty} t^{n} H_{E}^{n} h
$$

We write

$$
\chi_{j}=\frac{1}{\sqrt{2}} \exp \left(j \frac{\pi}{4} H_{E} \chi\right) \quad(j= \pm 1) .
$$

The following proposition plays an important role in the computation of capacities induced from the Hilbert transform.

Proposition 10. Let $T_{E}$ be the inverse operator of $I d_{E}-H_{E}^{2}$. Then, for any $h \in L^{2}(E)$,

(16) $T_{E} h=\frac{1}{2} h+\frac{1}{2}\left\{\chi_{1} H_{E}\left(h \chi_{-1}\right)-\chi_{-1} H_{E}\left(h \chi_{1}\right)\right\}$,

(17) $H_{E} T_{E} h=\frac{1}{2}\left\{\chi_{1} H_{E}\left(h \chi_{-1}\right)+\chi_{-1} H_{E}\left(h \chi_{1}\right)\right\}$.

Here are two lemmas necessary for the proof.

LEMMA 11 ([9]). $\chi_{t}=\frac{1}{\sqrt{1+t^{2}}} \exp \left\{\left(\int_{0}^{t} \frac{d s}{1+s^{2}}\right) H_{E} \chi\right\} \quad(|t|<1)$.

LEMMA 12. $h_{t}=\frac{1}{1+t^{2}} h+t \chi_{t} H_{E}\left(h \chi_{-t}\right) \quad\left(h \in L^{2}(E),|t|<1\right)$.

Proof. Note a formula

(18) $H_{E}\left(u H_{E} v+H_{E} u \cdot v\right)=H_{E} u \cdot H_{E} v-u v \quad\left(u, v \in L^{2}(E)\right)$. 
In fact, $H_{E}\left(u H_{E} v+H_{E} u \cdot v\right)+i\left(u H_{E} v+H_{E} u \cdot v\right)$ and $\left(H_{E} u+i u\right)\left(H_{E} v+i v\right)$ have analytic extensions to the upper half plane, and hence $g=H_{E}\left(u H_{E} v+H_{E}^{\prime} u \cdot v\right)-\left(H_{E} u \cdot H_{E} v-u v\right)$ has an analytic extension to the upper half plane. Analogously, $g$ has an analytic extension to the lower half plane. Thus $g=0$.

Let $h_{t} *$ denote the function in the right-hand side of the required equality. We have $\left(I d_{E}-t H_{E}\right) h_{t}=h$ and

$$
\begin{aligned}
\left(I d_{E}-t H_{E}\right) h_{t} *= & \frac{1}{1+t^{2}}\left(h-t H_{E} h\right) \\
& +t \chi_{t} H_{E}\left(h \chi_{-t}\right)-t^{2} H_{E}\left\{\chi_{t} H_{E}\left(h \chi_{-t}\right)\right\} .
\end{aligned}
$$

Lemma 11 shows that $\chi_{t} \chi_{-t}=1 /\left(1+t^{2}\right)$. By (18) and $\chi_{t}-t H_{E} \chi_{t}=\chi$, we have

$$
\begin{aligned}
& -t^{2} H_{E}\left\{\chi_{t} H_{E}\left(h \chi_{-t}\right)\right\}=t^{2} H_{E}\left(H_{E} \chi_{t} \cdot h \chi_{-t}\right)-t^{2} H_{E} \chi_{t} \cdot H_{E}\left(h \chi_{-t}\right)+t^{2} \chi_{t} h \chi_{-t} \\
= & t H_{E}\left\{\left(\chi_{t}-\chi\right) h \chi_{-t}\right\}-t\left(\chi_{t}-\chi\right) H_{E}\left(h \chi_{-t}\right)+\frac{t^{2}}{1+t^{2}} h \\
= & \frac{t}{1+t^{2}} H_{E} h-t \chi_{t} H_{E}\left(h \chi_{-t}\right)+\frac{t^{2}}{1+t^{2}} h .
\end{aligned}
$$

Substituting $-t^{2} H_{E}\left\{\chi_{t} H_{E}\left(h \chi_{-t}\right)\right\}$ by the last quantity in (20), we have, from (19), $\left(I d_{E}-t H_{E}\right) h_{t} *=h$. Thus $\left(I d_{E}-t H_{E}\right)\left(h_{t}-h_{t} *\right)=0$. Since the norm of $t H_{E}$ is less than $1, I d_{E}-t H_{E}$ is invertible, and hence $h_{t}=h_{t} *$. This completes the proof.

We now give the proof of Proposition 10. Since the adjoint operator of $H_{E}$ equals $-H_{E}, T_{E}$ exists. Let $T_{E, t}$ be the inverse operator of $I d_{E}-t^{2} H_{E}^{2}$ $(0<t<1)$. Then

$$
T_{E, t} h=\frac{1}{2}\left(h_{t}+h_{-t}\right)
$$

Lemma 12 shows that

$$
T_{E, t} h=\frac{1}{1+t^{2}} h+\frac{1}{2}\left\{t \chi_{t} H_{E}\left(h \chi_{-t}\right)-t \chi_{-t} H_{E}\left(h \chi_{t}\right)\right\} \quad(0<t<1) .
$$

Letting $t$ tend to 1 , we obtain (16). Lemma 12 shows that

$$
H_{E} T_{E, t} h=\frac{1}{2}\left(h_{t}-h_{-t}\right)=\frac{1}{2}\left\{t \chi_{t} H_{E}\left(h \chi_{-t}\right)+t \chi_{-t} H_{E}\left(h \chi_{t}\right)\right\} \text {. }
$$

Letting $t$ tend to 1 , we obtain (17). This completes the proof.

In this position, we show two lemmas used in the proof of our corollaries.

LEMMA 13.

(21) $H_{E} \chi_{j}=j(\chi,-\chi) \quad(j= \pm 1)$,

(22) $\mathcal{C}_{E} \chi_{j}(z)=j\left\{\Phi_{E}(z)^{\jmath}-1\right\} \quad(\jmath= \pm 1, z \in C-E)$, 
where $\Phi_{E}(z)=\exp \left\{\frac{1}{4} \int_{E} \frac{d x}{x-z}\right\}$.

Proof. Since $\chi_{t}-t H_{E} \chi_{t}=\chi \quad(|t|<1)$, we have (21). Since $\cos \frac{\pi}{4}=\sin \frac{\pi}{4}=$ $1 / \sqrt{2}$, we have

$$
\begin{aligned}
\lim _{\varepsilon \downarrow 0}\left\{\Phi_{E}(x \pm i \varepsilon)-1\right\} & =\chi_{1}(x)-1 \pm i \chi_{1}(x) \\
& =H_{E} \chi_{1}(x) \pm i \chi_{1}(x)=\lim _{\varepsilon \downarrow 0} c_{E} \chi_{1}(x \pm i \varepsilon) \text { a.e. on } E .
\end{aligned}
$$

Since both $\Phi_{E}(z)-1$ and $\mathcal{C}_{E} \chi_{1}(z)$ vanish at infinity, we have $\Phi_{E}(z)-1=\mathcal{C}_{E} \chi_{1}(z)$. In the same manner, we see that (22) holds for $j=-1$. This completes the proof.

LEMMA 14. We write

$$
X_{-}=\frac{1}{2}\left(\chi_{1}-\chi_{-1}\right), \quad X_{+}=\frac{1}{2}\left(\chi_{1}+\chi_{-1}\right) .
$$

For $d \in \boldsymbol{R}, d \neq 0$, we put

$$
\begin{aligned}
& A_{d}=\frac{1}{2}\left\{\Phi_{E}(d i)+\Phi_{E}(d i)^{-1}\right\}, \quad B_{d}=\frac{1}{2}\left\{\Phi_{E}(d i)-\Phi_{E}(d i)^{-1}\right\}, \\
& \sigma_{d}=\frac{1}{\pi} \int_{E} \frac{d x}{x^{2}+d^{2}} .
\end{aligned}
$$

Then

(23) $\mathcal{C}_{E} X_{-}(d i)=A_{d}-1$,

(24) $\mathcal{C}_{E} X_{+}(d i)=B_{d}$,

(25) $\frac{1}{\pi} \int_{E} \frac{1}{x^{2}+d^{2}} X_{-} d x=\frac{1}{2 d i}\left(A_{d}-\bar{A}_{d}\right)$,

(26) $\frac{1}{\pi} \int_{E} \frac{1}{x^{2}+d^{2}} X_{+} d x=\frac{1}{2 d i}\left(B_{d}-\bar{B}_{d}\right)$,

(27) $\frac{1}{\pi} \int_{E} \frac{1}{x^{2}+d^{2}} H_{E} X_{-} d x=\frac{1}{2 d i}\left(B_{d}-\bar{B}_{d}\right)-\sigma_{d}$,

(28) $\frac{1}{\pi} \int_{E} \frac{1}{x^{2}+d^{2}} H_{E} X_{+} d x=\frac{1}{2 d i}\left(A_{d}-\bar{A}_{d}\right)$.

Proof. By (22), we have (23) and (24). Since

$$
\frac{1}{x^{2}+d^{2}}=\frac{1}{2 d i}\left\{\frac{1}{x-d i}-\frac{1}{x+d i}\right\}, \quad \Phi_{E}(-d i)=\overline{\Phi_{E}(d i)},
$$

(23) and (24) yield (25) and (26), respectively. By (21), we have $H_{E} X_{-}=X_{+}-\chi$ 
and $H_{E} X_{+}=X_{-}$. Thus (25) and (26) yield (28) and (27), respectively. This completes the proof.

\section{Proof of corollaries.}

We now give the proof of Corollary 1. Our method shows a method of the construction of $\left(f_{0}, \phi_{0}\right)$. Let $E$ be a compact set on $\boldsymbol{R}$ and let $a \in \boldsymbol{C}-E$, Im $a \neq 0$. Translating and rotating the coordinate axes if necessary, we may assume that $a$ is purely imaginary and $\operatorname{Im} a>0$. Put $a=d i$. Then the required equality is rewritten as

(29) $\delta(E, d i,-d i)=2 \tan \theta_{d}, \quad \theta_{d}=\frac{1}{4} \int_{E} \frac{d}{x^{2}+d^{2}} d x$.

There exists a decreasing sequence $\left(E_{n}\right)_{n=1}^{\infty}$ of compact sets on $\boldsymbol{R}$ such that $E_{n} \in \mathcal{A}(n \geqq 1), E=\bigcap_{n=1}^{\infty} E_{n}$. Then we have $\delta(E, d i,-d i)=\lim _{n \rightarrow \infty} \delta\left(E_{n}, d i,-d i\right)$. Hence it is sufficient to prove (29) for $E_{n}$. From the beginning, we assume that $E \in A$. For the proof of (29), it is better to start from (12) than to use the formula in Theorem directly. Since $\bar{H}_{E}=H_{E},(12)$ is rewritten as

$$
h_{0}-\left(x^{2}+d^{2}\right) H_{E}\left(\frac{c_{0}+H_{E} h_{0}}{{ }^{2}+d^{2}}\right)=p_{0}(x-d i)+q_{0}
$$

Since

$$
\begin{aligned}
H_{E}\left(\frac{c_{0}+H_{E} h_{0}}{\cdot^{2}+d^{2}}\right)(x)= & \frac{1}{2 d \imath}\left\{H_{E}\left(\frac{c_{0}+H_{E} h_{0}}{\cdot-d_{\imath}}\right)(x)-H_{E}\left(\frac{c_{0}+H_{E} h_{0}}{\cdot+d i}\right)(x)\right\} \\
= & \frac{1}{2 d i(x-d i) \pi} p \cdot v \cdot \int_{E}\left\{\frac{1}{t-x}-\frac{1}{t-d i}\right\}\left(c_{0}+H_{E} h_{0}\right) d t \\
& -\frac{1}{2 d i(x+d i) \pi} p \cdot v \cdot \int_{E}\left\{\frac{1}{t-x}-\frac{1}{t+d i}\right\}\left(c_{0}+H_{E} h_{0}\right) d t \\
= & \frac{1}{2 d i(x-d i)}\left\{H_{E}\left(c_{0}+H_{E} h_{0}\right)(x)-\mathcal{C}_{E}\left(c_{0}+H_{E} h_{0}\right)(d i)\right\} \\
& -\frac{1}{2 d i(x+d i)}\left\{H_{E}\left(c_{0}+H_{E} h_{0}\right)(x)-\mathcal{C}_{E}\left(c_{0}+H_{E} h_{0}\right)(-d i)\right\} \\
= & \frac{1}{x^{2}+d^{2}} H_{E}\left(c_{0}+H_{E} h_{0}\right)(x)-\frac{1}{2 d i(x-d i)} \mathcal{C}_{E}\left(c_{0}+H_{E} h_{0}\right)(d i) \\
& +\frac{1}{2 d i(x+d i)} \mathcal{C}_{E}\left(c_{0}+H_{E} h_{0}\right)(-d i),
\end{aligned}
$$

we have

$$
\begin{aligned}
h_{0}-H_{E}^{2} h_{0}= & c_{0} H_{E} \chi-\frac{1}{2 d i} \mathcal{C}_{E}\left(c_{0}+H_{E} h_{0}\right)(d i)(x+d i) \\
& +\left\{p_{0}+\frac{1}{2 d i} \mathcal{C}_{E}\left(c_{0}+H_{E} h_{0}\right)(-d i)\right\}(x-d i)+q_{0},
\end{aligned}
$$


and hence

(31) $h_{0}=c_{0} T_{E} H_{E} \chi-r_{0} T_{E}(\cdot+d i)+\left(p_{0}+s_{0}\right) T_{E}(\cdot-d i)+q_{0} T_{E} \chi$,

where

$$
r_{0}=\frac{1}{2 d i} \mathcal{C}_{E}\left(c_{0}+H_{E} h_{0}\right)(d i), \quad s_{0}=\frac{1}{2 d i} \mathcal{C}_{E}\left(c_{0}+H_{E} h_{0}\right)(-d i)
$$

Since $H_{E}^{2 n+1}$ is an anti-symmetric operator, we have $\int_{E} H_{E}^{2 n+1} \chi d x=0(n \geqq 0)$. Hence

$$
\text { (32) } \frac{1}{\pi} \int_{E} \chi_{1} d x=\frac{1}{\pi} \int_{E} \chi_{-1} d x \quad\left(=t_{0}, \text { say }\right) \text {. }
$$

By (16), (21) and (32), we have

$$
\begin{aligned}
T_{E}(\cdot+d i)= & \frac{x+d i}{2}+\frac{1}{2}\left\{\chi_{1} H_{E}\left((\cdot+d i) \chi_{-1}\right)-\chi_{-1} H_{E}\left((\cdot+d i) \chi_{1}\right)\right\} \\
= & \frac{x+d i}{2}+\frac{1}{2 \pi}\left\{\chi_{1} p \cdot v \cdot \int_{E} \frac{t-x+x+d i}{t-x} \chi_{-1} d t\right. \\
& \left.-\chi_{-1} p \cdot v \cdot \int_{E} \frac{t-x+x+d i}{t-x} \chi_{1} d i\right\} \\
= & \frac{x+d i}{2}+\frac{1}{2}\left\{\chi_{1}\left(t_{0}+(x+d i) H_{E} \chi_{-1}\right)-\chi_{-1}\left(t_{0}+(x+d i) H_{E} \chi_{1}\right)\right\} \\
= & \frac{x+d i}{2}+\frac{1}{2}\left(\chi_{1}\left\{t_{0}+(x+d i)\left(-\chi_{-1}+1\right)\right\}-\chi_{-1}\left\{t_{0}+(x+d i)\left(\chi_{1}-1\right)\right\}\right) \\
= & t_{0} \frac{\chi_{1}-\chi_{-1}}{2}+(x+d i) \frac{\chi_{1}+\chi_{-1}}{2}=t_{0} X_{-}+(x+d i) X_{+} .
\end{aligned}
$$

Analogously,

Note that

$$
T_{E}(\cdot-d i)=t_{0} X_{-}+(x-d i) X_{+} .
$$

$$
\begin{aligned}
p_{0}-r_{0}+s_{0}= & \frac{1}{\pi} \int_{E} \frac{1}{x^{2}+d^{2}}\left(c_{0}+H_{E} h_{0}\right) d x-\frac{1}{2 d i \pi} \int_{E} \frac{1}{x-d i}\left(c_{0}+H_{E} h_{0}\right) d x \\
& +\frac{1}{2 d i \pi} \int_{E} \frac{1}{x+d i}\left(c_{0}+H_{E} h_{0}\right) d x=0 .
\end{aligned}
$$

Thus (31) yields that

$$
\begin{aligned}
h_{0} & =c_{0} X_{-}-r_{0}\left\{t_{0} X_{-}+(x+d i) X_{+}\right\}+\left(p_{0}+s_{0}\right)\left\{t_{0} X_{-}+(x-d i) X_{+}\right\}+q_{0} X_{+} \\
& =\left\{c_{0}+t_{0}\left(p_{0}-r_{0}+s_{0}\right)\right\} X_{-}+\left\{q_{0}-d i\left(p_{0}+r_{0}+s_{0}\right)\right\} X_{+}+\left(p_{0}-r_{0}+s_{0}\right) x / X_{+} \\
& =c_{0} X_{-}+\left\{q_{0}-d i\left(p_{0}+r_{0}+s_{0}\right)\right\} X_{+} \quad\left(=c_{0} X_{-}+c_{0}^{\prime} X_{+}, \text {say }\right) .
\end{aligned}
$$


We determine $c_{0}$ and $c_{0}^{\prime}$. By (23) and (24), the condition $c_{0}+\mathcal{C}_{E} h_{0}(d i)=1$ yields that

(34) $c_{0}+\left(A_{d}-1\right) c_{0}+B_{d} c_{0}^{\prime}=1$.

Condition $c_{0}+\mathcal{C}_{E} h_{0}(-d i)=1$ implies $\int_{E} h_{0} /\left(x^{2}+d^{2}\right) d x=0$, and hence, by (25) and (26),

(35) $\frac{1}{2 d i}\left(A_{d}-\bar{A}_{d}\right) c_{0}+\frac{1}{2 d i}\left(B_{d}-\bar{B}_{d}\right) c_{0}^{\prime}=0$.

Solving (34) and (35), we obtain

(36) $\quad c_{0}=-\frac{B_{d}-\bar{B}_{d}}{A_{d} \bar{B}_{d}-\bar{A}_{d} B_{d}}, \quad c_{0}^{\prime}=\frac{A_{d}-\bar{A}_{d}}{A_{d} \bar{B}_{d}-\bar{A}_{d} B_{d}}$.

Recall (11) and (15). By (27), (28) and (36), we obtain

$$
\begin{aligned}
\delta(E, d i,-d i) & =2 d p_{0}=\frac{2 d}{\pi} \int_{E}\left(c_{0}+H_{E} h_{0}\right) \frac{d x}{x^{2}+d^{2}} \\
& =2 d\left(c_{0} \sigma_{d}+\left\{\frac{B_{d}-\bar{B}_{d}}{2 d \imath}-\sigma_{d}\right\} c_{0}+\frac{A_{d}-\bar{A}_{d}}{2 d i} c_{0}^{\prime}\right) \\
& =\frac{1}{i} \frac{-\left(B_{d}-\bar{B}_{d}\right)^{2}+\left(A_{d}-\bar{A}_{d}\right)^{2}}{A_{d} \bar{B}_{d}-\bar{A}_{d} B_{d}} .
\end{aligned}
$$

Since

$$
\begin{aligned}
& \left(A_{d}-\bar{A}_{d}\right)^{2}-\left(B_{d}-\bar{B}_{d}\right)^{2}=\left(A_{d}^{2}-B_{d}^{2}\right)+\left(\bar{A}_{d}^{2}-\bar{B}_{d}^{2}\right)-2\left(\left|A_{d}\right|^{2}-\left|B_{d}\right|^{2}\right) \\
& =2\left\{1-\operatorname{Re} \Phi_{E}(d i) \Phi_{E}(-d i)^{-1}\right\}=2\left(1-\cos 2 \theta_{d}\right)=4 \sin ^{2} \theta_{d}, \\
& A_{d} \bar{B}_{d}-\bar{A}_{d} B_{d}=-i \operatorname{Im} \Phi_{E}(d i) \Phi_{E}(-d i)^{-1}=-i \sin 2 \theta_{d}=-2 i \sin \theta_{d} \cos \theta_{d},
\end{aligned}
$$

we have (29). This completes the proof.

From now we determine $\left(f_{0}, \phi_{0}\right)$. A simple calculation yields that

(37) $\phi_{0}(z)=\frac{2 d i}{z^{2}+d^{2}} \phi_{0}(z)^{2}=\frac{2 d i}{z^{2}+d^{2}}\left\{c_{0}+\mathcal{C}_{E} h_{0}(z)\right\}^{2}$

$$
=\frac{d i}{2 \cos ^{2} \theta_{d}} \frac{\left\{r_{d}^{-1} \Phi_{E}(z)+r_{d} \Phi_{E}(z)^{-1}\right\}^{2}}{z^{2}+d^{2}}
$$

where $r_{d}=\left|\Phi_{E}(d i)\right|$ and $\theta_{d}=\frac{1}{4} \int_{E} d /\left(x^{2}+d^{2}\right) d x$. Condition (5) shows that $\frac{1}{i} f_{0} \phi_{0} d z=\bar{\phi}_{0}|d z|$ a.e. on $\partial E$. Hence we have

$$
f_{0}(z) \phi_{0}(z)-f_{0}(\infty) \phi_{0}(\infty)=\frac{1}{2 \pi i} \int_{\partial E} \frac{1}{\zeta-z} f_{0} \phi_{0} d \zeta
$$




$$
=\frac{1}{2 \pi} \int_{\partial E} \frac{1}{\zeta-z} \bar{\phi}_{0}|d \zeta|
$$

which shows that

$$
f_{0}(z)=\frac{1}{\phi_{0}(z)}\left\{\frac{1}{2 \pi} \int_{\partial E} \frac{1}{\zeta-z} \bar{\phi}_{0}|d \zeta|-u_{0}\right\}
$$

for some constant $u_{0}$. We can compute $\frac{1}{2 \pi} \int_{\partial E} \frac{1}{\zeta-z} \bar{\phi}_{0}|d \zeta|$. Using (4), we determine $u_{0}$. Then

(38) $f_{0}(z)=\frac{r_{d}^{-1} \Phi_{E}(z)-r_{d} \Phi_{E}(z)^{-1}}{r_{d}^{-1} \Phi_{E}(z)+r_{d} \Phi_{E}(z)^{-1}}$.

Once $\left(f_{0}, \psi_{0}\right)$ is found, the proof of Corollary 1 is simplified. In fact, we have

$$
\begin{array}{ll}
\left|f_{0}(b)-f_{0}(a)\right| \leqq \delta(E, b, a) \leqq \sup _{|f| \leqq 1}|f(b)-f(a)| & \\
=\sup _{|f| \leqq 1} \frac{1}{2 \pi}\left|\int_{\partial E}\left\{\frac{1}{z-b}-\frac{1}{z-a}\right\} f d z\right|=\sup _{|f| \leqq 1} \frac{1}{2 \pi}\left|\int_{\partial E} f \phi_{0} d z\right| & \text { (by (3)) } \\
\leqq \frac{1}{2 \pi} \int_{\partial E}\left|\psi_{0}\right||d z|=\frac{1}{2 \pi} \int_{\partial E}\left|f_{0} \psi_{0}\right||d z| & \text { (by (4)) } \\
=\frac{1}{2 \pi i} \int_{\partial E} f_{0} \psi_{0} d z=\frac{1}{2 \pi i} \int_{\partial E} f_{0} d z=f_{0}(b)-f_{0}(a) & \text { (by (5)) }
\end{array}
$$

which gives (6). Computing $f_{0}(d i)-f_{0}(-d i)$, we obtain the required equality. Next we prove Corollary 2. Without loss of generality, we may assume that $E \in \mathcal{A}$ and $b>a$. We put

$$
\begin{aligned}
& f_{0}(z)=\varepsilon_{0} \frac{r_{a}^{-1} \Phi_{E}(z)-r_{b} \Phi_{E}(z)^{-1}}{r_{a}^{-1} \Phi_{E}(z)+r_{b} \Phi_{E}(z)^{-1}} \quad\left(\varepsilon_{0}=\operatorname{sign}\left\{r_{b} r_{a}^{-1}-1\right\}\right), \\
& \phi_{0}(z)=\frac{b-a}{4 \cosh ^{2} \theta_{a, b}} \frac{\left\{r_{a}^{-1} \Phi_{E}(z)+r_{b} \Phi_{E}(z)^{-1}\right\}^{2}}{(z-b)(z-a)}
\end{aligned}
$$

where

$$
\begin{aligned}
& \Phi_{E}(z)=\exp \left\{\frac{1}{4} \int_{E} \frac{d x}{x-z}\right\}, \quad r_{c}=\left|\Phi_{E}(c)\right| \quad(c=a, b), \\
& \theta_{a, b}=\frac{1}{4} \int_{E} \frac{(b-a) d x}{(x-b)(x-a)} .
\end{aligned}
$$

Note that $r_{b} \neq r_{a}$. We easily see that $\left(f_{0}, \phi_{0}\right)$ satisfies (3)-(5). Thus (6) shows that

$$
\delta(E, a, b)=f_{0}(b)-f_{0}(a)=\varepsilon_{0}\left\{\frac{r_{a}^{-1} r_{b}-1}{r_{a}^{-1} r_{b}+1}-\frac{1-r_{b} r_{a}^{-1}}{1+r_{b} r_{a}^{-1}}\right\}
$$




$$
\begin{aligned}
& =2\left|\frac{r_{b}^{1 / 2} r_{a}^{-1 / 2}-r_{b}^{-1 / 2} r_{a}^{1 / 2}}{r_{b}^{1 / 2} r_{a}^{-1 / 2}+r_{b}^{-1 / 2} r_{a}^{1 / 2}}\right| \\
& =2\left|\tanh \frac{\theta_{a, b}}{2}\right|=2 \tanh \left\{\frac{1}{8}\left|\int_{E} \frac{(b-a) d x}{(x-b)(x-a)}\right|\right\} .
\end{aligned}
$$

This completes the proof of Corollary 2 .

Remark 15. In the case where $\operatorname{Im} a \neq-\operatorname{Im} b$, the computation of $\delta(E, a, b)$ is complicated. An estimate from below is given by

$$
\delta(E, a, b) \geqq \max _{r \geq 0}\left|\frac{2 r\left\{\Phi_{E}(b) \Phi_{E}(a)^{-1}-\Phi_{E}(b)^{-1} \Phi_{E}(a)\right\}}{\left\{r \Phi_{E}(b)+\Phi_{E}(b)^{-1}\right\}\left\{r \Phi_{E}(a)+\Phi_{E}(a)^{-1}\right\}}\right| \quad(E \subset \boldsymbol{R}, a, b \in \boldsymbol{C}-E) .
$$

To see this, we take

$$
f_{r}(z)=\frac{r \Phi_{E}(z)-\Phi_{E}(z)^{-1}}{r \Phi_{E}(z)+\Phi_{E}(z)^{-1}} \quad(r \geqq 0) .
$$

Then $f_{r} \in H^{\infty}\left(E^{c}\right)$ and $\left\|f_{r}\right\|_{H^{\infty}} \leqq 1$. Hence

$$
\delta(E, a, b) \geqq \max _{r \geqq 0}\left|f_{r}(b)-f_{r}(a)\right|,
$$

which yields the required inequality.

We now deduce Corollary 3 from Corollary 1 . Let $E \subset \boldsymbol{T}$. We neglect the case where $E=\boldsymbol{T}$. (Evidently, $\delta(\boldsymbol{T}, 0, \infty)=2=2 \tan (|\boldsymbol{T}| / 4)$.) Hence, rotating the coordinate axes if necessary, we may assume that $E \not \supset-1$. Let $g(z)=$ $\frac{i(z-1)}{2(z+1)}$, then $g(0)=-i / 2$ and $g(\infty)=i / 2$. We have, with $F=\{g(z) ; z \in E\}$,

$$
\omega(-i / 2, F)=\omega^{*}(0, E)=\frac{1}{2 \pi}|E|,
$$

where $\omega(-i / 2, F)$ is the harmonic measure at $-i / 2$ of $F$ with respect to the lower half plane and $\omega^{*}(0, E)$ is the harmonic measure at 0 of $E$ with respect to the unit disk. Thus Corollary 1 and the conformal invariance show that

$$
\begin{aligned}
\delta(E, 0, \infty) & =\delta(F,-i / 2, i / 2)=2 \tan \left\{\frac{1}{4} \int_{F} \frac{1 / 2}{x^{2}+(1 / 4)} d x\right\} \\
& =2 \tan \left\{\frac{\pi}{4} \omega(-i / 2, F)\right\}=2 \tan (|E| / 8) .
\end{aligned}
$$

This completes the proof of Corollary 3.

\section{Application.}

In this section, we show some applications of our method. The AhlforsGarabedian method yields that, for $E \subset \boldsymbol{R}$ and $z \in \boldsymbol{C}-E$, 


$$
\begin{aligned}
& r(E)=\inf \left\{\frac{1}{\pi} \int_{E}\left(\left|1+H_{E} h\right|^{2}+|h|^{2}\right) d x ; h \in L^{2}(E)\right\}, \\
& c_{E}(z)=\inf \left\{\frac{1}{\pi} \int_{E}\left(\left|\frac{1}{x-z}+H_{E} h\right|^{2}+|h|^{2}\right) d x ; h \in L^{2}(E)\right\} .
\end{aligned}
$$

For $a \in \boldsymbol{C}$, a compact set $E$ on $\boldsymbol{R}$ and a measure $\nu$ supported in $\boldsymbol{C}-E$, we define a capacity by

$$
\gamma(E, a, \nu)=\inf \left\{\frac{1}{\pi} \int_{E}\left(\left|a+\mathcal{C} \nu+H_{E} h\right|^{2}+|h|^{2}\right) d x ; h \in L^{2}(E)\right\},
$$

where $c_{\nu}(x)=\frac{1}{\pi} \int \frac{1}{z-x} d \nu(z)$. Notice that $\gamma(E)=\gamma(E, 1,0)$ and $c_{E}(z)=\gamma\left(E, 0, \pi \delta_{z}\right)$, where $\delta_{z}$ is the Dirac measure supported at $z$. We show

PROPOSITION 16.

$$
\begin{aligned}
\gamma(E, a, \nu)= & \frac{1}{4}|a|^{2}|E|+\operatorname{Re} \frac{2 \bar{a}}{\pi} \int \sinh \left\{\frac{1}{4} \int_{E} \frac{d x}{x-z}\right\} d \nu(z) \\
& +\frac{1}{\pi^{2}} \iint \frac{1}{z-\bar{\zeta}} \sinh \left\{\frac{1}{4} \int_{E} \frac{(z-\bar{\zeta}) d x}{(x-z)(x-\bar{\zeta})}\right\} d \nu(z) \overline{d \nu(\zeta),}
\end{aligned}
$$

where the integrand in the last term means $\frac{1}{4} \int_{E}(x-z)^{-2} d x$ if $z=\bar{\zeta}$.

Proof. There exists $k_{0} \in L^{2}(E)$ which attains $\gamma(E, a, \nu)$. A variational method shows that, for any $h \in L^{2}(E)$,

$$
\frac{1}{\pi} \int_{E}\left\{\left(a+C \nu+H_{E} k_{0}\right) H_{E} \bar{h}+k_{0} \bar{h}\right\} d x=0,
$$

and hence $\left(I d_{E}-H_{E}^{2}\right) k_{0}=H_{E}(a+\mathcal{C} \nu)$, i.e., $k_{0}=T_{E} H_{E}(a+\mathcal{C} \nu)$. Thus

$$
\begin{aligned}
\gamma(E, a, \nu)= & \frac{1}{\pi} \int_{E}\left(a+C_{\nu}+H_{E} k_{0}\right) \overline{(a+\mathcal{C} \nu)} d x \\
& +\frac{1}{\pi} \int_{E}\left\{\left(a+\mathcal{C} \nu+H_{E} k_{0}\right) H_{E} \bar{k}_{0}+k_{0} \bar{k}_{0}\right\} d x \\
= & \left.\frac{1}{\pi} \int_{E}\left(I d_{E}+H_{E} T_{E} H_{E}\right)(a+\mathcal{C} \nu) \cdot \overline{(a+\mathcal{C} \nu}\right) d x \\
= & \left.\frac{1}{\pi} \int_{E} T_{E}(a+\mathcal{C} \nu) \cdot \overline{(a+\mathcal{C} \nu}\right) d x \\
= & \frac{|a|^{2}}{\pi} \int_{E} T_{E} \chi d x+\operatorname{Re}\left\{\frac{2 \bar{a}}{\pi} \int_{E} T_{E}(\mathcal{C} \nu) d x\right\}+\frac{1}{\pi} \int_{E} T_{E}\left(\mathcal{C}_{\nu}\right) \cdot \overline{\mathcal{C}_{\nu}} d x \\
= & J_{1}+J_{2}+J_{3}, \quad \operatorname{say.}
\end{aligned}
$$


(Here $\operatorname{Re} \zeta$ is the real part of $\zeta$.) Since $\frac{1}{\pi} \int_{E} T_{E} \chi d x=|E| / 4$, we have $J_{1}=$ $|a|^{2}|E| / 4$ (cf. [9]). Equality (16) and Lemma 13 show that, for $z \in C-E$,

$$
\text { (39) } \begin{aligned}
T_{E}\left(\frac{1}{\cdot-z}\right)(x) & =\frac{1}{2(x-z)}+\frac{1}{2} \sum_{j= \pm 1} j \chi_{j}(x) H_{E}\left(\frac{\chi_{-\jmath}}{\cdot-z}\right)(x) \\
& =\frac{1}{2(x-z)}+\frac{1}{2} \sum_{j= \pm 1} j \frac{\chi_{j}(x)}{x-z}\left\{H_{E} \chi_{-j}(x)-\mathcal{C}_{E} \chi_{-j}(z)\right\} \\
& =\frac{1}{2(x-z)}+\frac{1}{2} \sum_{\jmath= \pm 1} j \frac{\chi_{\jmath}(x)}{x-z}(-j)\left\{\chi_{-j}(x)-\Phi_{E}(z)^{-j}\right\} \\
& =\frac{\Phi_{E}(z)^{-1}}{2} \frac{\chi_{1}(x)}{x-z}+\frac{\Phi_{E}(z)}{2} \frac{\chi_{-1}(x)}{x-z} .
\end{aligned}
$$

Using (22) and (39), we have, with $K=$ (the support of $\nu$ ),

$$
\text { (40) } \begin{aligned}
J_{2} & =\operatorname{Re}\left\{\frac{2 \bar{a}}{\pi^{2}} \int_{K}\left(\int_{E} T_{E}\left(\frac{1}{\cdot-z}\right)(x) d x\right) d \nu(z)\right\} \\
& =\operatorname{Re}\left\{\sum_{\jmath= \pm 1} \frac{\bar{a}}{\pi} \int_{K} \Phi_{E}(z)^{-\jmath} \mathcal{C}_{E} \chi_{j}(z) d \nu(z)\right\} \\
& =\operatorname{Re}\left\{\frac{\bar{a}}{\pi} \int_{K}\left(\Phi_{E}(z)-\Phi_{E}(z)^{-1}\right) d \nu(z)\right\} \\
& =\operatorname{Re}\left\{\frac{2 \bar{a}}{\pi} \int_{K} \sinh \left(\frac{1}{4} \int_{E} \frac{d x}{x-z}\right) d \nu(z)\right\} .
\end{aligned}
$$

Equalities (16), (22) and (39) show that, for $z, \zeta \in C-E, z \neq \bar{\zeta}$,

$$
\text { (41) } \begin{aligned}
\frac{1}{\pi} & \int_{E} T_{E}\left(\frac{1}{\cdot-z}\right)(x) \frac{1}{x-\bar{\zeta}} d x \\
& =\frac{1}{2 \pi} \sum_{\jmath= \pm 1} \Phi_{E}(z)^{-j} \int_{E} \frac{\chi_{j}(x)}{(x-z)(x-\bar{\zeta})} d x \\
& =\frac{1}{2(z-\bar{\zeta})} \sum_{j= \pm 1} \Phi_{E}(z)^{-\jmath}\left\{\mathcal{C}_{E} \chi_{j}(z)-\mathcal{C}_{E} \chi_{j}(\bar{\zeta})\right\} \\
& =\frac{1}{2(z-\bar{\zeta})} \sum_{\jmath= \pm 1} \Phi_{E}(z)^{-\jmath} j\left\{\Phi_{E}(z)^{\jmath}-\Phi_{E}(\bar{\zeta})^{j}\right\} \\
& =\frac{1}{2(z-\bar{\zeta})}\left\{\Phi_{E}(z) \Phi_{E}(\bar{\zeta})^{-1}-\Phi_{E}(z)^{-1} \Phi_{E}(\bar{\zeta})\right\} \\
& =\frac{1}{z-\bar{\zeta}} \sinh \left\{\frac{1}{4} \int_{E} \frac{(z-\bar{\zeta}) d x}{(x-z)(x-\bar{\zeta})}\right\} .
\end{aligned}
$$

Since the first quantity in (41) is continuous in $C-E$ as a function of $z$, we have 


$$
\frac{1}{\pi} \int_{E} T_{E}\left(\frac{1}{\cdot-z}\right)(x) \frac{1}{x-z} d x=\frac{1}{4} \int_{E} \frac{d x}{(x-z)^{2}}
$$

Thus

(42) $J_{3}=\frac{1}{\pi^{3}} \int_{K} \int_{K}\left\{\int_{E} T_{E}\left(\frac{1}{\cdot-z}\right)(x) \frac{1}{x-\bar{\zeta}} d x\right\} d \nu(z) \overline{d \nu(\zeta)}$

$$
=\frac{1}{\pi^{2}} \int_{K} \int_{K} \frac{1}{z-\bar{\zeta}} \sinh \left\{\frac{1}{4} \int_{E} \frac{(z-\bar{\zeta}) d x}{(x-z)(x-\bar{\zeta})}\right\} d \nu(z) \overline{d \nu(\zeta)} \text {. }
$$

Consequently, by $J_{1}=|a|^{2}|E| / 4$, (40) and (42), we obtain the required equality. This completes the proof.

For $E \in \mathscr{F}, \hat{K}_{E}(z, \bar{\zeta})$ denotes the Szegö kernel function with respect to $H^{2}\left(E^{c}\right)$, i.e.,

$$
f(z)=\int_{\partial E} \hat{K}_{E}(z, \bar{\zeta}) f|d \zeta| \quad\left(f \in H^{2}\left(E^{c}\right)\right) .
$$

The Szegö kernel function is closely related to $\gamma(E)$ and $c_{E}(z)$. We here note the following proposition (cf. [2]).

Proposition 17. Let $E \subset \boldsymbol{R}$. Then

$$
\begin{aligned}
\hat{K}_{E}(z, \bar{\zeta})= & \frac{2}{\pi|E|} \cosh \left\{\frac{1}{4} \int_{E} \frac{d x}{x-z}\right\} \cosh \left\{\frac{1}{4} \int_{E} \frac{d x}{x-\bar{\zeta}}\right\} \\
& +\frac{1}{2 \pi(z-\bar{\zeta})} \sinh \left\{\frac{1}{4} \int_{E} \frac{(z-\bar{\zeta}) d x}{(x-z)(x-\bar{\zeta})}\right\} .
\end{aligned}
$$

Proof. We begin by showing that

(43) $\hat{K}_{E}(z, \bar{z})=\frac{1}{2 \pi \gamma(E)}\left|\phi_{E}(z)\right|+\frac{1}{2 \pi} c_{E}(z) \quad(z \in \boldsymbol{C}-E)$,

where $\phi_{E}$ is the Garabedian function with respect to $\gamma(E)$, i.e., the function in $H^{1}\left(E^{c}\right)$ satisfying $\phi_{E}(\infty)=1$ and $\gamma(E)=\frac{1}{2 \pi} \int_{\partial E}\left|\psi_{E}\right||d z|[5$, p. 19$]$.

It is known the $\sqrt{\psi_{E}}\left(=\phi_{E}\right.$, say) is single-valued and

(44) $\phi_{E}(z)=2 \pi \gamma(E) \hat{K}(z, \bar{\infty}), \quad \hat{K}_{E}(\infty, \bar{\infty})=\frac{1}{2 \pi \gamma(E)} \quad[5$, p. 22$]$.

For any $z \in \boldsymbol{C}-E$, there exists a pair $\left(f_{z}, \phi_{z}\right)$ of functions such that

(45) $\begin{cases}f_{z} \in H^{\infty}\left(E^{c}\right), & (\cdot-z) \phi_{z} \in H^{2}\left(E^{c}\right), \\ \phi_{z}(\infty)=0, & \lim _{\zeta \rightarrow z}(\zeta-z) \phi_{z}(\zeta)=1,\end{cases}$

(46) $\left|f_{z}\right|=1, \quad \frac{1}{i} f_{z} \phi_{z} d \zeta=\bar{\phi}_{z}|d \zeta|$ a. e. on $\partial E$ ([3, Chap. VII $\left.]\right)$. 
Then we have

(47) $c_{E}(z)=f_{z}^{\prime}(z)$.

For any $f \in H^{2}\left(E^{c}\right)$, we have, by (45) and (46),

$$
\begin{aligned}
\int_{\partial E} \hat{K}_{E}(z, \bar{\zeta}) f|d \zeta| & =f(z)=f(\infty)+\frac{1}{2 \pi i} \int_{\partial E} \frac{f(\zeta)-f(\infty)}{\zeta-z} d \zeta \\
& =f(\infty)+\frac{1}{2 \pi i} \int_{\partial E} \phi_{z}\{f(\zeta)-f(\infty)\} d \zeta \\
& =\left\{1-\frac{1}{2 \pi i} \int_{\partial E} \phi_{z} d \zeta\right\} f(\infty)+\frac{1}{2 \pi} \int_{\partial E} \overline{f_{z} \phi_{z}} f|d \zeta| \\
& =\int_{\partial E}\left\{c_{z} \hat{K}_{E}(\infty, \bar{\zeta})+\frac{1}{2 \pi} \overline{f_{z} \phi_{z}}\right\} f|d \zeta|
\end{aligned}
$$

where $c_{z}=1-\frac{1}{2 \pi i} \int_{\partial E} \phi_{z} d \zeta$. Hence

$$
\text { (48) } \hat{K}_{E}(z, \bar{\zeta})=c_{z} \hat{K}_{E}(\infty, \bar{\zeta})+\frac{1}{2 \pi} \overline{f_{z}(\bar{\zeta}) \phi_{z}(\zeta)} .
$$

Letting $\zeta$ tend to infinity in (48), we have, by (44),

$$
\hat{K}_{E}(z, \bar{\infty})=c_{z} \hat{K}_{E}(\infty, \bar{\infty})=\frac{c_{z}}{2 \pi \gamma(E)} .
$$

Letting $\zeta$ tend to $z$ in (48), we have, by (47),

$$
\begin{aligned}
\hat{K}_{E}(z, \bar{z}) & =c_{z} \hat{K}_{E}(\infty, \bar{z})+\frac{1}{2 \pi} \overline{f_{z}^{\prime}(z)} \\
& =2 \pi \gamma(E)\left|\hat{K}_{E}(z, \bar{\infty})\right|^{2}+\frac{1}{2 \pi} c_{E}(z)=\frac{1}{2 \pi \gamma(E)}\left|\phi_{E}(z)\right|+\frac{1}{2 \pi} c_{E}(z) .
\end{aligned}
$$

Thus (43) holds. It is known that

$$
\text { (49) } \phi_{E}(z)=\cosh \left\{\frac{1}{4} \int_{E} \frac{d x}{x-z}\right\} \quad \text { ([9]). }
$$

Let

$$
\begin{aligned}
F(z, \bar{\zeta})= & \hat{K}_{E}(z, \bar{\zeta})-\frac{1}{2 \pi \gamma(E)} \phi_{E}(z) \overline{\phi_{E}(\zeta)} \\
& -\frac{1}{\pi} \int_{E} T_{E}\left(\frac{1}{\cdot-z}\right)(x) \frac{1}{x-\zeta} d x \quad(z, \zeta \in C-E)
\end{aligned}
$$

Since

$$
c_{E}(z)=\frac{1}{\pi} \int_{E} T_{E}\left(\frac{1}{\cdot-z}\right)(x) \frac{1}{x-\bar{z}} d x
$$


(43) shows that $F(z, \bar{z})=0(z \in \boldsymbol{C}-E)$, which yields $F(z, \bar{\zeta})=0(z, \zeta \in \boldsymbol{C}-E)$, by the theorem of identity. Thus (41), (49) and $\gamma(E)=|E| / 4$ yield the required equality. This completes the proof.

The following extremum problem is the special case of the Pick-Nevanlinna interpolation problem:

$$
\eta(E, z)=\sup \left\{|f(z)| ; f \in H^{1}\left(E^{c}\right),\|f\|_{H^{\infty}} \leqq 1, f(\infty)=0\right\} \quad(z \in C-E) .
$$

Evidently, $\left|f_{E}(z)\right| \leqq \eta(E, z)$ and the equality does not hold in general, where $f_{E}$ is the Ahlfors function with respect to $\gamma(E)$, i.e., $f_{E} \in H^{\infty}\left(E^{c}\right),\left\|f_{E}\right\|_{H^{\infty}} \leqq 1, \gamma(E)=$ $f_{E}^{\prime}(\infty)[5$, p. 18]. If $E \subset \boldsymbol{R}$, then the Ahlfors-Garabedian method shows that

$$
\eta(E, z)=\inf \left\{\frac{1}{\pi} \int_{E}\left(\left|1+H_{E} h\right|^{2}+|h|^{2}\right) \frac{d x}{|x-z|} ; h \in L^{2}(E)\right\} .
$$

Thus our method enable us to compute $\eta(E, z)$ in the case of $E \subset \boldsymbol{R}$. 'A calculation shows that

(50) $\eta(E, z)=\frac{1}{\pi} \int_{E} k_{0} d x$

with the solution $k_{0} \in L^{2}(E)$ of

$$
\left(I d_{E}-M_{0} H_{E} M_{0}^{-1} H_{E}\right) k_{0}=\tau_{0} \quad\left(\tau_{0}(x)=\frac{1}{|x-z|}, M_{0}=M_{\tau_{0}}\right)
$$

In fact, by a variational method, we obtain

$$
\eta(E, z)=\frac{1}{\pi} \int_{E}\left(1+H_{E} k_{0} *\right) \tau_{0} d x
$$

with the solution $k_{0} *$ of $\left(M_{0}-H_{E} M_{0} H_{E}\right) k_{0} *=H_{E} \tau_{0}$. Let $k_{0}=\left(1+H_{E} k_{0} *\right) \tau_{0}$. Then $k_{0}$ satisfies (51), which gives (50). If $z \in \boldsymbol{R}$ satisfies $z<\min \{x ; x \in E\}$, then (50) and (51) yield that

$$
\eta(E, z)=\frac{\Phi_{E}(z)-\Phi_{E}(z)^{-1}}{\Phi_{E}(z)+\Phi_{E}(z)^{-1}}=f_{E}(z) .
$$

The extremum pair with respect to $\eta(E, z)$ is given by

$$
\left(\left(\Phi_{E}-\Phi_{\bar{E}}^{-1}\right) /\left(\Phi_{E}+\Phi_{E}^{-1}\right),\left(\Phi_{E}+\Phi_{\bar{E}}^{-1}\right)^{2} /\{4(\zeta-z)\}\right) .
$$

Our method works for more general extremum problems. As an example, we study

$$
\delta(E, \boldsymbol{A}, \boldsymbol{W})=\sup \left\{\left|\sum_{k=1}^{n} w_{k} f\left(a_{k}\right)\right| ; f \in H^{\infty}\left(E^{c}\right),\|f\|_{H^{\infty}} \leqq 1\right\},
$$

where $E \subset \boldsymbol{C}, \boldsymbol{A}=\left\{a_{k}\right\}_{k=1}^{n} \subset E^{c}\left(a_{k} \neq a_{\jmath}, k \neq j\right)$ and $\boldsymbol{W}=\left\{w_{k}\right\}_{k=1}^{n} \subset \boldsymbol{C}$. In the special case, we can compute $\delta(E, \boldsymbol{A}, \boldsymbol{W})$. We show 
Proposition 18. For $E \subset \boldsymbol{R}$ and $d>0$, let $\left(f_{0}, \phi_{0}\right)$ be the pair defined by (37) and (38). Suppose that $\boldsymbol{A}=\left\{a_{k}\right\}_{k=1}^{2 n} \subset \boldsymbol{C}-E$ and $\boldsymbol{W}=\left\{w_{k}\right\}_{k=1}^{2 n}(n \geqq 2)$ satisfy $a_{1}=\bar{a}_{2}$ $=d i, a_{2 \jmath-1}=\bar{a}_{2 \jmath},(2 \leqq j \leqq n), \quad \sum_{k=1}^{2 n} w_{k}=0$ and $w_{k}$ equals the residue of $\psi_{A}$ at $a_{k}$ $1 \leqq k \leqq 2 n$, where

$$
\phi_{A}(z)=\prod_{k=3}^{2 n} \frac{a_{1}-a_{k}}{z-a_{k}} \phi_{0}(z)
$$

Then

$$
\delta(E, \boldsymbol{A}, \boldsymbol{W})=\left|\sum_{k=1}^{2 n} w_{k} f_{0}\left(a_{k}\right)\right|
$$

Proof. Let

$$
f_{A}(z)=e^{-i \theta} A f_{0}(z), \quad \theta_{A}=\arg \prod_{k=3}^{2 n}\left(a_{1}-a_{k}\right) .
$$

Then the pair $\left(f_{A}, \phi_{A}\right)$ satisfies

$$
\left|f_{A}\right|=1, \quad \frac{1}{i} f_{A} \psi_{A} d z=\left|\psi_{A}\right||d z| \text { a.e. on } \partial E \text {. }
$$

Since

$$
\sum_{k=1}^{2 n} w_{k}=0, \quad \lim _{z \rightarrow \infty} z \psi_{A}(z)=0, \quad \lim _{z \rightarrow a_{k}}\left(z-a_{k}\right) \phi_{A}(z)=w_{k} \quad(1 \leqq k \leqq 2 n),
$$

we have

$$
\begin{aligned}
\left|\sum_{k=1}^{2 n} w_{k} f_{0}\left(a_{k}\right)\right| & =\left|\sum_{k=1}^{2 n} w_{k} f_{A}\left(a_{k}\right)\right| \\
& \leqq \delta(E, \boldsymbol{A}, \boldsymbol{W})=\sup _{|f| \leqq 1} \frac{1}{2 \pi}\left|\int_{\partial E} \sum_{k=1}^{2 n} \frac{w_{k}}{z-a_{k}} f d z\right| \\
& =\sup _{|f| \leqq 1} \frac{1}{2 \pi}\left|\int_{\partial E} \phi_{A} f d z\right| \leqq \frac{1}{2 \pi} \int_{\partial E}\left|\phi_{\boldsymbol{A}}\right||d z| \\
& =\frac{1}{2 \pi} \int_{\partial E}\left|\phi_{A} f_{\boldsymbol{A}}\right||d z|=\frac{1}{2 \pi i} \int_{\partial E} \boldsymbol{\psi}_{A} f_{\boldsymbol{A}} d z \\
& =\sum_{k=1}^{2 n} w_{k} f_{\boldsymbol{A}}\left(a_{k}\right)=\left|\sum_{k=1}^{2 n} w_{k} f_{0}\left(a_{k}\right)\right|,
\end{aligned}
$$

which gives the required equality. This completes the proof.

\section{REFERENCES}

[1] L. Ahlfors, Bounded analytic functions, Duke Math. J. 14 (1947), 1-11.

[2] W. BAKER II, Kernel functions on domains with hyperelliptic double, Trans. Amer. Math. Soc. 231 (1977), 339-347.

[3] S. Bergman, The kernel function and conformal mapping, Amer. Math. Soc. Colloq. Publ., New York, 1950. 
[4] P. Garabedian, Schwarz's lemma and the Szegö kernel function, Trans. Amer. Math. Soc. 143 (1949), 187-200.

[5] J. Garnett, Analytic capacity and measure, Lecture Notes in Math. 297, SpringerVerlag, Berlin, 1972.

[6] D. A. HEJHAL, Linear extremal problems for analytic functions with interior side conditions, Ann. Acad. Sci. Fenn. 586 (1974), 5-36.

[7] P.D. LAx, Reciprocal extremal problems in function theory, Comm. Pure Appl. Math. 13 (1955), 437-453.

[8] T. Murai, A real variable method for the Cauchy transform, and analytic capacity, Lecture Notes in Math. 1307, Springer-Verlag, Berlin, 1988.

[9] T. Mural, The power $3 / 2$ appearing in the estimate of analytic capacity, Pacific J. Math. 143 (1990), 313-340.

[10] L. Sario and K. Oikawa, Capacity functions, Springer-Verlag, Berlin, 1969.

[11] Ch. Pommerenke, Über die analytische Kapazität, Arch. Math. 11 (1960), 270-277.

Department of Mathematics

School of Science

NAGOYA UNIVERSITY

NAGOYA, 464

JAPAN 